Original Research Paper

\title{
Some Cubic Transmuted Exponentiated Pareto-1 Distribution
}

\author{
Hussein Eledum \\ Department of Statistics, University of Tabuk, Tabuk, KSA, Saudi Arabia \\ Department of Applied Statistics, University of Shendi, Shendi, Sudan
}

\author{
Article history \\ Received: 05-06-2020 \\ Revised: 22-06-2020 \\ Accepted: 11-07-2020 \\ Email: heledum@yahoo.com
}

\begin{abstract}
In this study, we introduce two new generalized versions of the Exponentiated Pareto-I distribution called (CTEP-I-G) and (CTEP-I-R). Statistical properties of the two distributions such as reliability function, hazard function, moments and moment generating function are studied. Models parameters are estimated by the maximum likelihood method. Finally, an application of CTEP-I-G and CTEP-I-R distributions to two real datasets and compared with some distributions based on exponentiated Pareto-I distribution is illustrated. The applications suggest that the CTEPI-G performs better than CTEP-I-R.
\end{abstract}

Keywords: Pareto Distribution, Transmuted Family, Moments, Survival Function, Maximum Likelihood

\section{Introduction}

In order to obtain more flexible statistical models, several methods for generalizing of the well-known distributions have been widely used. For examples, (Gupta et al., 1998) proposed the exponentiated family of distributions to model the failure time data. Several authors use (Gupta et al., 1998) method to propose new distributions for examples see (Gupta and Kundu, 1999; 2001; 2007). Eugene et al. (2002) introduced a general class of distributions generated from the logit of the beta random variable. Another family of distributions, known as Kw-G distributions, proposed by (Cordeiro and de Castro, 2011) by using cdf of the Kumaraswamy distribution. Shaw and Buckley (2009) suggested a new generalization method called transmution map. According to (Shaw and Buckley, 2009) the cumulative distribution function of the Quadratic Ranking Transformation Map (QRTM) is:

$$
F(x)=(1+\lambda) G(x)-\lambda G^{2}(x) ;|\lambda| \leq 1
$$

where, $G(x)$ is the Cumulative Distribution Function (cdf) of the base distribution. Observe that, when $\lambda=0$, the new distribution turns out to the base one. This method has been further studied and extended by many authors see for examples (Aryal and Tsokos, 2009; 2011; Nofal et al., 2017; Alizadeh et al., 2016; Merovci et al., 2016).

Al-Kadim (2018) introduced generalized formula for transmuted distribution proposed by Shaw and Buckley (2009), the cdf and pdf of the Cubic Ranking Transformation Map (CRTM) respectively are:

$$
F(x)=G(x)\left[(1+\lambda)-2 \lambda G(x)+\lambda G^{2}(x)\right] ;|\lambda| \leq 1
$$

and:

$$
f(x)=g(x)\left[(1+\lambda)-4 \lambda G(x)+3 \lambda G^{2}(x)\right] ;|\lambda| \leq 1
$$

This method used by (Al-Kadim and Mohammed, 2017) to develop cubic transmuted Weibull distribution and utilized by (Ansari and Eledum, 2018; 2020) to introduce the Cubic Transmuted Pareto distribution and cubic transmuted exponentiated Pareto-I respectively. Another two classes of Cubic Transmuted distributions with two transmuted parameters have been developed, one by (Granzotto et al., 2017) the other by (Rahman et al., 2018b). The cdf and pdf of the Granzottoa formula are as follows:

$$
F(x)=\lambda_{1} G(x)+\left(\lambda_{2}-\lambda_{1}\right) G^{2}(x)+\left(1-\lambda_{2}\right) G^{3}(x)
$$

and:

$$
f(x)=g(x)\left[\lambda_{1}+2\left(\lambda_{2}-\lambda_{1}\right) G(x)+3\left(1-\lambda_{2}\right) G^{2}(x)\right]
$$

with, $\lambda_{1}, \in[0,1]$ and $\lambda_{2} \in[-1,1]$.

This method used by many authors to generalized a various of well-known distributions for examples (Granzotto et al., 2017; Celik, 2018). Concerning to (Rahman et al., 2018b) method, the cdf and pdf are given respectively by:

$$
F(x)=\left(1+\omega_{1}\right) G(x)+\left(\omega_{2}-\omega_{1}\right) G^{2}(x)-\omega_{2} G^{3}(x)
$$

and: 


$$
f(x)=g(x)\left[1+\omega_{1}+2\left(\omega_{2}-\omega_{1}\right) G(x)-3 \omega_{2} G^{2}(x)\right]
$$

with $\omega_{1}, \omega_{2} \in[-1,1]$ and $-2 \leq \omega_{1}+\omega_{2} \leq 1$.

This formula used to propose some new distributions for examples Pareto distribution (Rahman et al., 2018a) and Weibull distribution (Rahman et al., 2019). In this article we use cubic transmuted distributions proposed by (Granzotto et al., 2017) of Equation (4) and by (Rahman et al., 2018b) of Equation (6) to propose two new generalized versions of the Exponentiated Pareto-I distribution which called Cubic Transmuted Exponentiated Pareto-I-G (CTEP-I-G) and Cubic Transmuted Exponentiated Pareto-I-R (CTEP-I-R) respectively. Some statistical properties are studied and the models parameters are estimated using maximum likelihood method. Moreover, an application of two models CTEP-I-G and CTEP-I-R to two real datasets and compared with some distributions based on exponentiated Pareto-I distribution is illustrated.

The rest of this paper is structured as follows: The first version of the Cubic Transmuted Exponentiated Pareto-1-G (CTEP-1-G) is presented in section 2, investigating the density and cumulative functions, survival and hazard functions, moreover, some statistical properties such as moments and moment generating function are studied, this section also provides parameters estimation of CTEP-1-G distribution. Section 3 pertains to the second version of the Cubic Transmuted Exponentiated Pareto-1-R (CTEP-1-R). An application of the two proposed distributions CTEP-1- G and CTEP-1-R to two real datasets for the purpose of illustration is conducted in section 4 . Finally, section 5 gives some concluding remarks.

The new two models is motivated because it exhibits a bimodal right skewed distribution as illustrated in Fig. 1a and 3a. The justification for the practicality of the two new generalized models is based on its ability for modelling the failure times data as illustrated in section 4 .

\section{Some Special Cases}

It can be easily see that the cubic transmuted family of distributions proposed by (Al-Kadim, 2018) in Equation (2) turned out to be a special case of the cubic transmuted family distribution proposed by:

- Granzotto et al. (2017) of Equation (4) for $\lambda_{1}=1+\lambda$ and $\lambda_{2}=1-\lambda$

- Rahman et al. (2018b) of Equation (6) for $\omega_{1}=-\omega_{2}=\lambda$

Moreover, the quadratic transmuted family of distributions of (Shaw and Buckley, 2009) in Equation (1), reduces to the cubic transmuted family distribution introduced by:

- Granzotto et al. (2017) of Equation (4) for $\lambda_{1}=$ $1+\lambda$ and $\lambda_{2}=1$
- $\quad$ Rahman et al. (2018b) of Equation (6) for $\omega_{1}=\lambda$ and $\omega_{2}=0$

Further, the cubic transmuted family distribution introduced by (Rahman et al., 2018b) in Equation (6) is a special case of the family proposed by (Granzotto et al., 2017) of Equation (4) for $\omega_{1}=\lambda_{1}-1$ and $\omega_{2}=\lambda_{2}-1$.

\section{Exponentiated Pareto Distribution-1}

The Exponential Pareto-1 distribution (EP-1) proposed by (Nadarajah, 2005) taking the logarithm of the classical Pareto random variable. Let $X$ be a random variable with the exponentiated Pareto-1 distribution. The Probability Density Function (pdf) and the Cumulative Distribution Function (cdf) are defined, respectively, as:

$$
G(x)=1-k^{a} e^{-a x}=1-z ; x \geq \ln k, a, k>0
$$

and:

$g(x)=a k^{a} e^{-a x}=a z$

where, $z=k^{a} e^{-a x}, k$ is the (necessarily positive) minimum possible value of $X$ and $a$ is a shape parameter:

$$
\begin{aligned}
& E(X)=\frac{1+a \log k}{a} \\
& \operatorname{Var}(X)=\frac{1}{a^{2}} \\
& M(t)=\frac{a k^{t}}{a-t} \\
& \text { Skewness }(X)=2 \\
& \text { Kurtosis }(X)=9
\end{aligned}
$$

\section{Cubic Transmuted Exponentiated Pareto-1- G Distribution (CTEP-1-G)}

This section pertains to the first version of the Cubic Transmuted Exponentiated Pareto-1-G (CTEP-1-G) using the cubic transmuted distributions proposed by Granzotto et al. (2017). Discussing the Probability Density Function (PDF), Cumulative Distribution Function (CDF), survival and hazard functions, some statistical properties and parameters estimates.

\section{Density and Cumulative Function for CTEP-1-G Distribution}

\section{Theorem 1}

Let $X$ be a random variable with the CTEP-1-G distribution. The Cumulative Distribution Function (CDF) and the Probability Density Function (PDF) are defined, respectively, as: 


$$
\begin{aligned}
& F(x)=\left(1-k^{a} e^{-a x}\right)\left[1+\left(\lambda_{1}+\lambda_{2}-2\right) k^{a} e^{-a x}\right. \\
& \left.+\left(1-\lambda_{2}\right) k^{2 a} e^{-2 a x}\right] \\
& =(1-z)\left[1+\left(\lambda_{1}+\lambda_{2}-2\right) z+\left(1-\lambda_{2}\right) z^{2}\right] \\
& \quad a, k>0, x \geq \ln k, \lambda_{1} \geq 0, \lambda_{2} \in[-1,1]
\end{aligned}
$$

and:

$$
\begin{aligned}
& f(x)=a k^{a} e^{-a x}\left[3-\lambda_{2}-\lambda_{1}+2\left(2 \lambda_{2}+\lambda_{1}-3\right) k^{a} e^{-a x}\right. \\
& +3\left(1-\lambda_{2}\right) k^{2 a} e^{-2 a x} \\
& =a z\left[3-\lambda_{2}-\lambda_{1}+2\left(2 \lambda_{2}+\lambda_{1}-3\right) z+3\left(1-\lambda_{2}\right) z^{2}\right]
\end{aligned}
$$

where, $a, k$ are a shape and location parameters respectively and $\lambda_{1}, \lambda_{2}$ are transmuted parameters. We can see that the CTEP-1-G distribution given in Equation (10) reduces to the EP-1 distribution given in Equation (8), for $\lambda_{1}=\lambda_{2}=1$.

\section{Proof}

The proof is straightforward. Equation (10) is obtained by substituting Equation (8) into Equation (4) and performing some algebra calculations and Equation (11) is gotten from differentiating Equation (10) with respect to $x$.

\section{Remark 1}

Consider the random variable $Z=u(X)=k^{a} e^{-a x} \Rightarrow X=\varpi(Z)=\frac{a \ln k-\ln z}{a}$ then for $(x=$ $\ln k) \Rightarrow(z=1)$ and for $(x \rightarrow \infty) \Rightarrow(z \rightarrow 0)$ also $\frac{d x}{d z}=\frac{-1}{a z}$

Using Remark 1, Equation (11) can be rewritten in term of the random variable $Z$ as follows:

$$
\begin{aligned}
& f(z)=f[\varpi(z)] \frac{d x}{d z} \\
& =a z\left[3-\lambda_{2}-\lambda_{1}+2\left(2 \lambda_{2}+\lambda_{1}-3\right) z+3\left(1-\lambda_{2}\right) z^{2}\right]\left(\frac{-1}{a z}\right) \\
& =(-1)\left[3-\lambda_{2}-\lambda_{1}+2\left(2 \lambda_{2}+\lambda_{1}-3\right) z+3\left(1-\lambda_{2}\right) z^{2}\right] \\
& \text { if } z \in[0,1]
\end{aligned}
$$

\section{Lemma 1}

The limit of CTEP-1-G density as $x \rightarrow \ln k$ is $a \lambda_{1}$ and as $x \rightarrow \infty$ is 0 .

\section{Proof}

The proof is straightforward.

\section{Lemma 2}

The limit of CTEP-1-G distribution function as $x \rightarrow$ $\ln k$ is 0 and the limit as $x \rightarrow \infty$ is 1 .

\section{Proof}

The proof is straightforward.

\section{Lemma 3}

$f(x)$ of Equation (11) is a probability density function.

Proof

To prove $f(x)$ is a pdf, we need to prove $f(x) \geq 0$ and $\int_{\ln k}^{\infty} f(x) d x=1$.

From Lemma $1 \lim _{x \rightarrow \ln k} f(x)=a \lambda_{1}$ and $\lim _{x \rightarrow \infty} f(x)=0$, since $a, k>0$ and $\lambda_{1} \in[0,1]$ then $a \lambda_{1} \geq 0$. It follows that $f(x) \geq 0$.

The proof of $\int_{\ln k}^{\infty} f(x) d x=1$ is equivalent to prove $\int_{1}^{0} f(z) d z=1$ and $-\int_{0}^{1} f(z) d z=1$, using Equation (12) we get:

$$
\begin{aligned}
& \int_{0}^{1} f(z) d z=\int_{0}^{1}\left[3-\lambda_{2}-\lambda_{1}+2\left(2 \lambda_{2}+\lambda_{1}-3\right) z+3\left(1-\lambda_{2}\right) z^{2}\right] d z \\
& =\left(3-\lambda_{2}-\lambda_{1}\right) \int_{0}^{1} d z+2\left(2 \lambda_{2}+\lambda_{1}-3\right) \int_{0}^{1} z d x \\
& +3\left(1-\lambda_{2}\right) \int_{0}^{1} z^{2} d x \\
& =\left.\left(3-\lambda_{2}-\lambda_{1}\right)(z)\right|_{0} ^{1}+\left.\left(2 \lambda_{2}+\lambda_{1}-3\right)\left(z^{2}\right)\right|_{0} ^{1} \\
& +\left.\left(1-\lambda_{2}\right)\left(z^{3}\right)\right|_{0} ^{1} \\
& =3-\lambda_{2}-\lambda_{1}+2 \lambda_{2}+\lambda_{1}-3+1-\lambda_{2}=1
\end{aligned}
$$

Therefore, Lemma is Proved.

Some of possible shapes of the pdf and cdf of CTEP$1-\mathrm{G}$ for selected values of $\lambda_{1}$ and $\lambda_{2}$ setting $a=0.5$ and $k$ $=1$ are illustrated in Fig. $1 \mathrm{a}$ and $1 \mathrm{~b}$ respectively, note that the pdf and cdf of the base distribution EP-1 are shown as a solid black curves.

\section{Survival and Hazard Function}

The survival function is defined as $s(x)=1-F(x)$ and for the CTEP-1-G distribution is given as:

$$
S(x)=1-(1-z)\left[1+\left(\lambda_{1}+\lambda_{2}-2\right) z+\left(1-\lambda_{2}\right) z^{2}\right]
$$

The hazard function is defined as $h(x)=\frac{f(x)}{s(x)}$ and for the CTEP-1-G distribution is given as:

$$
h(x)=\frac{a z\left[3-\lambda_{2}-\lambda_{1}+2\left(2 \lambda_{2}+\lambda_{1}-3\right) z+3\left(1-\lambda_{2}\right) z^{2}\right]}{1-(1-z)\left[1+\left(\lambda_{1}+\lambda_{2}-2\right) z+\left(1-\lambda_{2}\right) z^{2}\right]}
$$

where, $z=k^{a} e^{-a x}$. Figures $2 \mathrm{a}$ and $2 \mathrm{~b}$ illustrate the behavior of the survival and hazard functions of CTEP$1-\mathrm{G}$ for selected values of $\lambda_{1}$ and $\lambda_{2}$ setting $a=0.5$ and $k=$ 1 , where $s(x)$ and $h(x)$ of EP-1 distribution are demonstrated as a solid black curves. Note that $h(x)$ for EP-1 distribution is a constant function, that is $h(x)=a$ if $\lambda_{1}=\lambda_{2}=1$. 


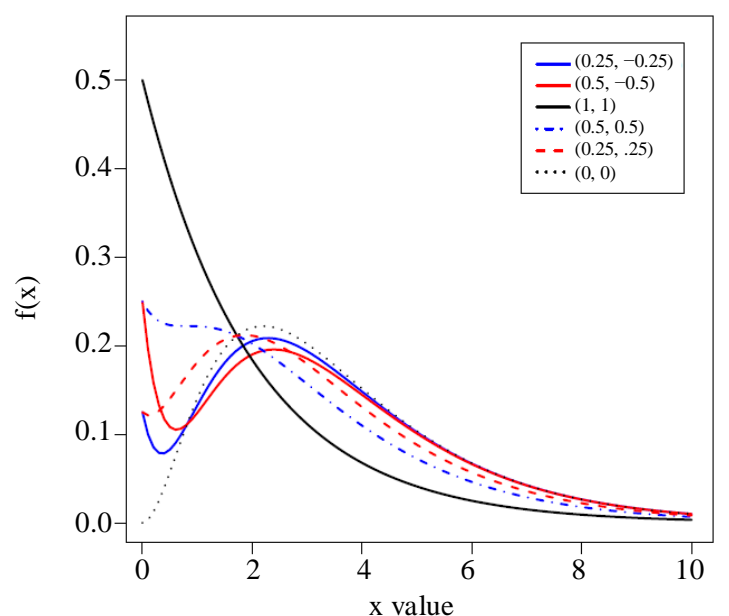

(a)

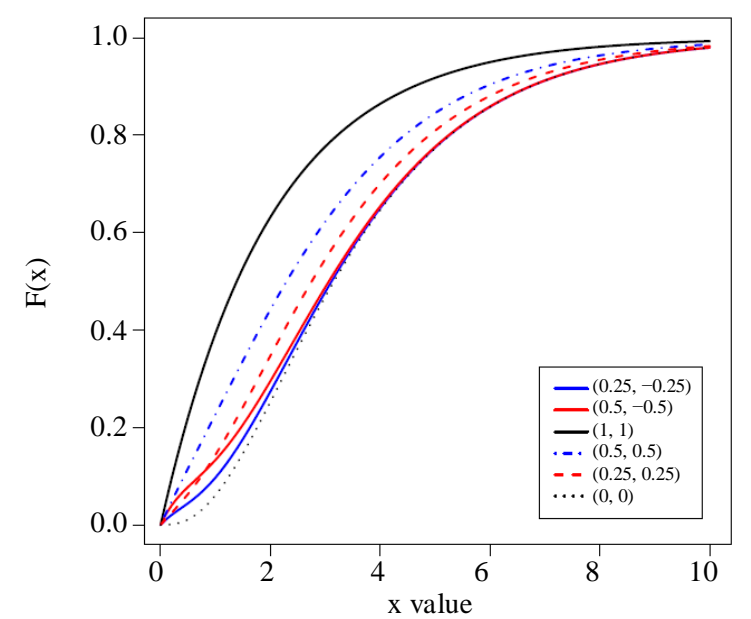

(b)

Fig. 1: The pdf and cdf of CTEP-1-G for different values of parameter $\lambda_{1}$ and $\lambda_{2}$ setting $a=0.5$ and $k=1$

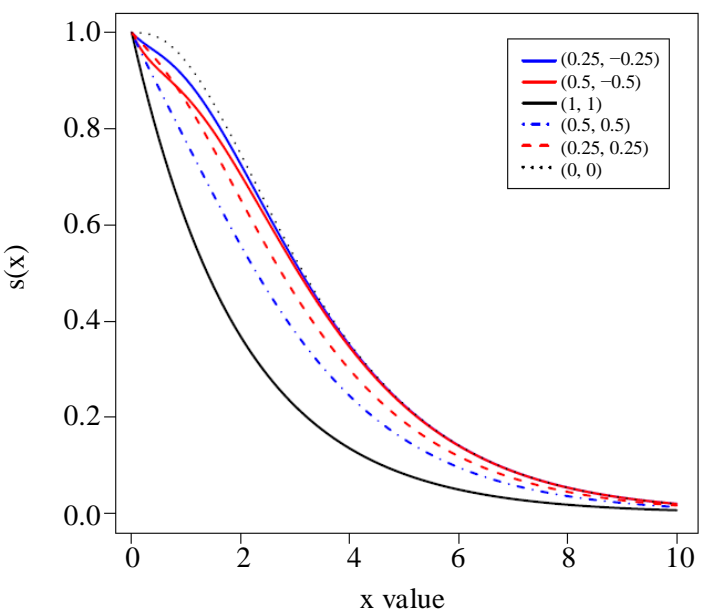

(a)

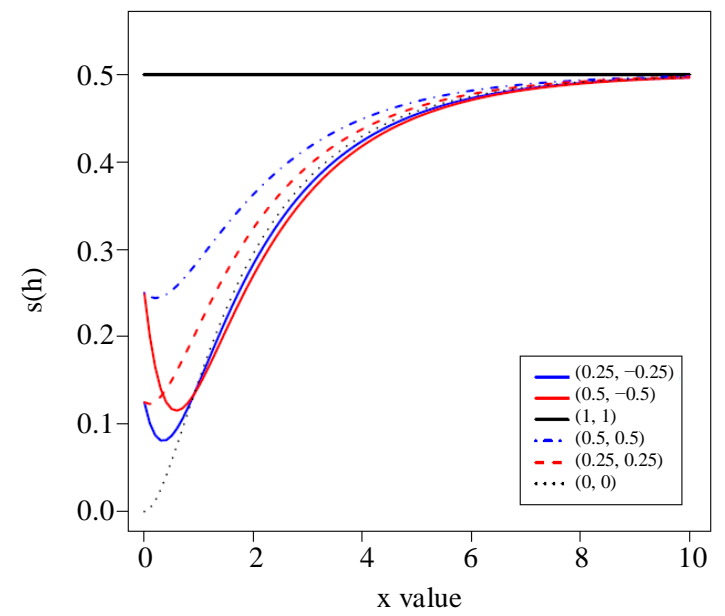

(b)

Fig. 2: The $s(x)$ and $h(x)$ of CTEP-1-G for different values of parameter $\lambda_{1}$ and $\lambda_{2}$ setting $a=0.5$ and $k=1$

\section{Statistical Properties}

In this subsection, some statistical properties for CTEP$1-\mathrm{G}$ distribution are explained. Including moments, moment generating function and order statistics.

\section{Moments}

\section{Theorem 2}

If $X$ is a random variable having the CTEP-1-G then the $r$ th moment of $X$ about the origin is:

$$
\begin{aligned}
& E\left(X^{r}\right)=\frac{k^{a}}{a^{r}}\left\{\left(3-\lambda_{2}-\lambda_{1}\right) \Gamma(r+1, a \ln k)\right. \\
& +\frac{k^{a}}{2^{r}}\left(2 \lambda_{2}+\lambda_{1}-3\right) \Gamma(r+1,2 a \ln k) \\
& \left.+\frac{k^{2 a}}{3^{r}}\left(1-\lambda_{2}\right) \Gamma(r+1,3 a \ln k)\right\}
\end{aligned}
$$

\section{Proof}

We know that:

$$
E\left(X^{r}\right)=\int_{-\infty}^{\infty} x^{r} f(x) d x
$$

Substitute $f(x)$ in above equation by its value in Equation (11) to get:

$$
\begin{aligned}
& E\left(X^{r}\right)=\int_{\ln k}^{\infty} x^{r}\left\{a k ^ { a } e ^ { - a x } \left[3-\lambda_{2}-\lambda_{1}+2\left(2 \lambda_{2}+\lambda_{1}-3\right)\right.\right. \\
& \left.\left.\times k^{a} e^{-a x}+3\left(1-\lambda_{2}\right) k^{2 a} e^{-2 a x}\right]\right\} d x \\
& =a k^{a}\left(3-\lambda_{2}-\lambda_{1}\right) \int_{\ln k}^{\infty} x^{r} e^{-a x} d x+2 a k^{2 a}\left(2 \lambda_{2}+\lambda_{1}-3\right) \\
& \times \int_{\ln k}^{\infty} x^{r} e^{-2 a x} d x+3 a k^{3 a}\left(1-\lambda_{2}\right) \int_{\ln k}^{\infty} x^{r} e^{-3 a x} d x \\
& =a k^{a}\left(3-\lambda_{2}-\lambda_{1}\right) I_{1}+2 a k^{2 a}\left(2 \lambda_{2}+\lambda_{1}-3\right) I_{2} \\
& +3 a k^{3 a}\left(1-\lambda_{2}\right) I_{3}
\end{aligned}
$$


where,

$$
I_{1}=\int_{\ln k}^{\infty} x^{r} e^{-a x} d x, I_{2}=\int_{\ln k}^{\infty} x^{r} e^{-2 a x} d x
$$

and

$$
I_{3}=\int_{\ln k}^{\infty} x^{r} e^{-3 a x} d x \text { now: }
$$

$$
\begin{aligned}
& I_{1}=\int_{\ln k}^{\infty} x^{r} e^{-a x} d x=1 / a^{r+1} \int_{a \ln k}^{\infty} u^{(r+1)-1} e^{-u} d x \\
& =1 / a^{r+1} \Gamma(r+1, a \ln k)
\end{aligned}
$$

where, $u=a x, d x=d u / a$ it follows that for $x=\ln k$ then $u$ $=a \ln k$ and for $x=\infty$ then $\mathrm{u}=\infty$ similarly $I_{2}=1 /(2 a)^{r+1}$ $\Gamma(r+1,2 a \ln k)$ and $I_{3}=1 /(3 a)^{r+1} \Gamma(r+1,3 a \ln k)$ by performing some algebra calculations we get:

$$
\begin{aligned}
& E\left(X^{r}\right)=\frac{k^{a}}{a^{r}}\left\{\left(3-\lambda_{2}-\lambda_{1}\right) \Gamma(r+1, a \ln k)+\frac{k^{a}}{2^{r}}\left(2 \lambda_{2}+\lambda_{1}-3\right)\right. \\
& \left.\times \Gamma(r+1,2 a \ln k)+\frac{k^{2 a}}{3^{r}}\left(1-\lambda_{2}\right) \Gamma(r+1,3 a \ln k)\right\}
\end{aligned}
$$

\section{Moments Generating Function}

\section{Theorem 3}

If $X$ is a random variable having the CTEP-1-G then the moments generating function of $X$ is:

$$
\begin{aligned}
& M_{X}(t)=\frac{a k^{t}\left(3-\lambda_{2}-\lambda_{1}\right)}{a-t} \\
& +\frac{2 a k^{t}\left(2 \lambda_{2}+\lambda_{1}-3\right)}{2 a-t}+\frac{3 a k^{t}\left(1-\lambda_{2}\right)}{3 a-t}
\end{aligned}
$$

where, $a-t>0,2 a-t>0$ and $3 a-t>0$.

Further, the mean and variance of the distribution are given respectively as:

$$
E(X)=\ln k+\frac{11-2 \lambda_{2}-3 \lambda_{1}}{6 a}
$$

and:

$$
\operatorname{Var}(X)=\frac{49+12 \lambda_{1}-9 \lambda_{1}^{2}-12 \lambda_{1} \lambda_{2}-4 \lambda_{2}^{2}}{36 a^{2}}
$$

\section{Proof}

We know that:

$$
M_{X}(t)=E\left(e^{t x}\right)=\int_{-\infty}^{\infty} e^{t x} f(x) d x
$$

Substitute $f(x)$ in above equation by its value in Equation (11) to get:
$M_{X}(t)=\int_{\ln k}^{\infty} e^{t x}\left\{a k^{a} e^{-a x}\left[3-\lambda_{2}-\lambda_{1}+2\left(2 \lambda_{2}+\lambda_{1}-3\right)\right.\right.$

$\left.\left.\times k^{a} e^{-a x}+3\left(1-\lambda_{2}\right) k^{2 a} e^{-2 a x}\right]\right\} d x$

$=a k^{a}\left(3-\lambda_{2}-\lambda_{1}\right) \int_{\ln k}^{\infty} e^{-(a-t) x} d x+2 a k^{2 a}\left(2 \lambda_{2}+\lambda_{1}-3\right)$

$\times \int_{\ln k}^{\infty} e^{-(2 a-t) x} d x+3 a k^{3 a}\left(1-\lambda_{2}\right) \int_{\ln k}^{\infty} e^{-(3 a-t) x} d x$

$=a k^{a}\left(3-\lambda_{2}-\lambda_{1}\right) W_{1}+2 k^{2 a}\left(2 \lambda_{2}+\lambda_{1}-3\right) W_{2}+3 k^{3 a}\left(1-\lambda_{2}\right) W_{3}$

where,

$$
W_{1}=\int_{\ln k}^{\infty} e^{-(a-t) x} d x, W_{2}=\int_{\ln k}^{\infty} e^{-(2 a-t) x} d x \text { and }
$$

$W_{3} \int_{\ln k}^{\infty} e^{-(3 a-t) x} d x$ now:

$$
\begin{aligned}
& W_{1}=\int_{\ln k}^{\infty} e^{-(a-t) x} d x=\frac{-1}{a-t}\left[e^{-(a-t) x}\right]_{\ln k}^{\infty} \\
& =\frac{k^{-(a-t)}}{a-t}
\end{aligned}
$$

Similarly, $\quad W_{2}=\frac{k^{-(2 a-t)}}{2 a-t} \quad$ and $\quad W_{3}=\frac{k^{-(3 a-t)}}{3 a-t} \quad$ by performing some algebra calculations we get:

$$
M_{X}(t)=\frac{a k^{t}\left(3-\lambda_{2}-\lambda_{1}\right)}{a-t}+\frac{2 a k^{t}\left(2 \lambda_{2}+\lambda_{1}-3\right)}{2 a-t}+\frac{3 a k^{t}\left(1-\lambda_{2}\right)}{3 a-t}
$$

The mean and variance have been obtained by differentiating Equation (14) $i$ times $(i=1,2)$ with respect to $t$ and setting $t=0$. Table 1 and 2 show the mean and variance of CTEP-1-G for various combinations of model parameters respectively.

From Table 1 it is observed that, holding the transmuted parameters $\lambda_{1}$ and $\lambda_{2}$ constants, as the shape parameter $a$ increases the mean decreases. On the other hand, holding $a$ constant, as the transmuted parameters $\lambda_{1}$ and $\lambda_{2}$ increase the mean also decreases. From Table 2 it is clear that, keeping the shape parameter $a$ and transmuted parameters $\lambda_{1}$ constants, as the transmuted parameter $\lambda_{2}$ increases the variance decreases. Further, keeping $a$ constant, as $\lambda_{1}$ increases the variance increases this for negative values of $\lambda_{2}$, while, for $\lambda_{2}>0$ the variance decreases.

\section{Order Statistics}

Let $X_{1}, X_{2}, \ldots, X_{n} \sim$ iid $C T E P-1-\mathrm{G}\left(a, k, \lambda_{1}, \lambda_{2}\right)$. Then the pdf of the $X(n)$ is given by:

$$
\begin{aligned}
& f_{(i)}(x)=n f(x)\left(\begin{array}{c}
n-1 \\
j-1
\end{array}\right) F(x)^{j-1}[1-F(x)]^{n-j} \\
& =a n z\left(\begin{array}{c}
n-1 \\
j-1
\end{array}\right)\left[3-\lambda_{2}-\lambda_{1}+2\left(2 \lambda_{2}+\lambda_{1}-3\right) z+3\left(1-\lambda_{2}\right) z^{2}\right] \\
& \times(1-z)\left[1+\left(\lambda_{1}+\lambda_{2}-2\right) z+\left(1-\lambda_{2}\right) z^{2}\right]^{j-1} \\
& \times\left[1-(1-z)\left[1+\left(\lambda_{1}+\lambda_{2}=2\right) z+\left(1-\lambda_{2}\right) z^{2}\right]\right]^{n-j}
\end{aligned}
$$


where, $z=k^{a} \mathrm{e}^{-a x}, x \geq \ln k, a, k>0$. Using $j=1$ and $j=n$ we get the pdf of smallest $X_{1: n}$ and largest $X_{n: n}$ order statistic respectively:

$$
\begin{aligned}
& f_{(1)}(x)=\operatorname{anz}\left[3-\lambda_{2}-\lambda_{1}+2\left(2 \lambda_{2}+\lambda_{1}-3\right) z+3 \mid\left(1-\lambda_{2}\right) z^{2}\right] \\
& \times\left[1-(1-z)\left[1+\left(\lambda_{1}+\lambda_{2}-2\right) z+\left(1-\lambda_{2}\right) z^{2}\right]\right]^{n-1} \\
& f_{(n)}(x)=\operatorname{anz}\left[3-\lambda_{2}-\lambda_{1}+2\left(2 \lambda_{2}+\lambda_{1}-3\right) z+3\left(1-\lambda_{2}\right) z^{2}\right] \\
& \times(1-z)\left[1+\left(\lambda_{1}+\lambda_{2}-2\right) z+\left(1-\lambda_{2}\right) z^{2}\right]^{n-1}
\end{aligned}
$$

\section{Parameters Estimation}

This subsection discusses the Maximum Likelihood Estimation (MLE) for parameters of CTEP-1-G distribution.

Let $X_{1}, X_{2}, \ldots, X_{n}$ be a random sample of size $n$ from CTEP-1-G distribution. Then the likelihood function is given by:

$$
\begin{aligned}
& L=\prod_{i=1}^{n} f\left(x_{i}\right) \\
& =\prod_{i=1}^{n} a k^{a} e^{-a x_{i}}\left[3-\lambda_{2}-\lambda_{1}+2\left(2 \lambda_{2}+\lambda_{1}-3\right) k^{a} e^{-a x_{i}}\right. \\
& \left.+3\left(1-\lambda_{2}\right) k^{2 a} e^{-2 a x_{i}}\right] \\
& =a^{n} k^{a n} e^{-a \sum_{i=1}^{n} x_{i}} \prod_{i=1}^{n}\left[3-\lambda_{2}-\lambda_{1}+2\left(2 \lambda_{2}+\lambda_{1}-3\right)\right. \\
& \left.\times k^{a} e^{-a x_{i}}+3\left(1-\lambda_{2}\right) k^{2 a} e^{-2 a x_{i}}\right]
\end{aligned}
$$

so, the $\log$ likelihood function is:

$$
\begin{aligned}
& \ln k=n \ln a+a n \ln k-a \sum_{i=1}^{n} x_{i}+\sum_{i=1}^{n} \ln \left[3-\lambda_{2}-\lambda_{1}+2\left(2 \lambda_{2}+\lambda_{1}-3\right)\right. \\
& \left.\times k^{a} e^{-a x_{i}}+3\left(1-\lambda_{2}\right) k^{2 a} e^{-2 a x_{i}}\right]
\end{aligned}
$$

Since $x \in(\ln k, \infty)$, the MLE of $\ln k$ is the firstorder statistic $x_{(1)}$. The log-likelihood can be maximized either directly or by solving the non-linear likelihood equations obtained by differentiating Equation (15). The components of the score vector are given by:

\begin{tabular}{|c|c|c|c|c|c|c|c|c|c|c|}
\hline & \multicolumn{3}{|l|}{$\begin{array}{l}a=1 \\
\lambda_{1}\end{array}$} & \multicolumn{3}{|l|}{$\begin{array}{l}a=3 \\
\lambda_{1}\end{array}$} & \multicolumn{3}{|l|}{$\begin{array}{l}a=5 \\
\lambda_{1}\end{array}$} \\
\hline & & 0.25 & 0.5 & 1 & 0.25 & 0.5 & 1 & 0.25 & 0.5 & 1 \\
\hline \multirow[t]{4}{*}{$\overline{\lambda_{2}}$} & -0.5 & 1.4427 & 1.5208 & 1.5833 & 0.1603 & 0.1690 & 0.1759 & 0.0577 & 0.0608 & 0.0633 \\
\hline & 0 & 1.4288 & 1.4653 & 1.4444 & 0.1588 & 0.1628 & 0.1605 & 0.0572 & 0.0586 & 0.0578 \\
\hline & 0.5 & 1.3594 & 1.3542 & 1.2500 & 0.1510 & 0.1505 & 0.1389 & 0.0544 & 0.0542 & 0.0500 \\
\hline & 1 & 1.2344 & 1.1875 & 1.0000 & 0.1372 & 0.1319 & 0.1111 & 0.0494 & 0.0475 & 0.0400 \\
\hline
\end{tabular}

$$
\begin{aligned}
\frac{\partial \ln k}{\partial a}=\frac{n}{a}+n \ln k-\sum_{i=1}^{n} x_{i}+2 k^{a} \\
\times \sum_{i=1}^{n} \frac{\left(\ln k-x_{i}\right)\left[2 \lambda_{2}+\lambda_{1}+3 k^{a} e^{-a x_{i}}\left(1-\lambda_{2}\right)\right] e^{-a x_{i}}}{R_{1}} \\
\frac{\partial \ln k}{\partial \lambda_{1}}=\sum_{i=1}^{n} \frac{\left(2 k^{a} e^{-a x_{i}}-1\right)}{R_{1}} \\
\frac{\partial \ln k}{\partial \lambda_{2}}=\sum_{i=1}^{n} \frac{\left[k^{a}\left(4-3 k^{a} e^{-a x_{i}}\right) e^{-a x_{i}}-1\right]}{R_{1}}
\end{aligned}
$$

where, $\quad R_{1}=\left[3-\lambda_{2}-\lambda_{1}+2\left(2 \lambda_{2}+\lambda_{1}-3\right) \quad k^{a} e^{-a x_{i}}+3\left(1-\lambda_{2}\right)\right.$ $\left.k^{2 a} e^{-2 a x_{i}}\right]$.

Now setting, $\frac{\partial \ln k}{\partial a}=0, \frac{\partial \ln k}{\partial \lambda_{1}}=0$ and $\frac{\partial \ln k}{\partial \lambda_{2}}=0$ and solving the nonlinear system of equations, the maximum likelihood estimates $\hat{\theta}=\left(\hat{a}, \hat{\lambda}_{1}, \hat{\lambda}_{2}\right)$ of $\theta=\left(a, \lambda_{1}, \lambda_{2}\right)$ is obtained.

Table 1: Mean of CTEP-1-G distribution

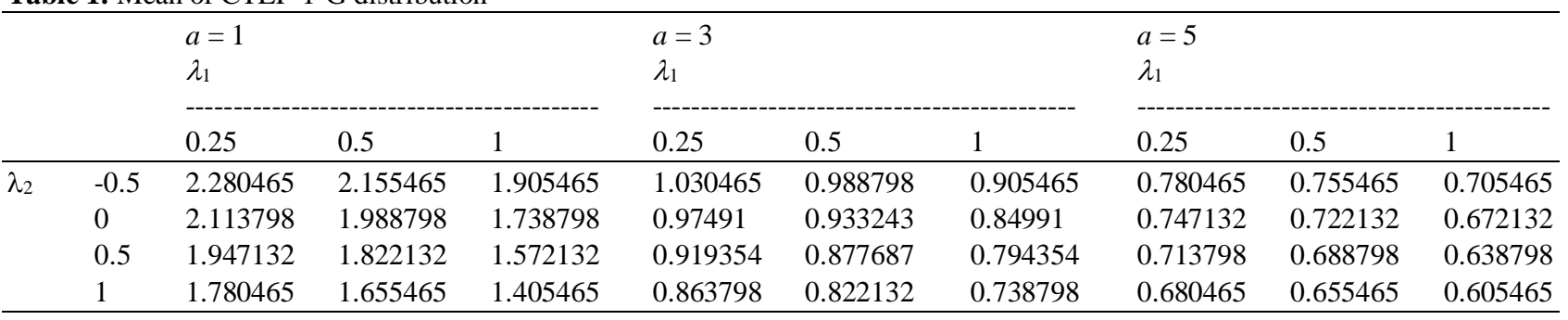

Table 2: Variance of CTEP-1-G distribution 


\section{Cubic Transmuted Exponentiated Pareto-1- R Distribution (CTEP-1-R)}

This section pertains to the second version of the Cubic Transmuted Exponentiated Pareto-1-R (CTEP-1R) using the cubic transmuted distributions proposed by (Rahman et al., 2018b), studding the Probability Density Function (pdf), Cumulative Distribution Function (CDF), survival and hazard functions, some statistical properties and parameters estimates.

\section{Density and Cumulative Function for CTEP-1-R Distribution}

\section{Theorem 4}

Let $X$ be a random variable with the CTEP-1-R distribution. The Cumulative Distribution Function (CDF) and the Probability Density Function (PDF) are defined, respectively, as:

$$
\begin{aligned}
& F(x)=\left(1-k^{a} e^{-a x}\right)\left[1+\left(\omega_{1}+\omega_{2}\right) k^{a} e^{-a x}-\omega_{2} k^{2 a} e^{-2 a x}\right] \\
& =(1-z)\left[1+\left(\omega_{1}+\omega_{2}\right) z-\omega_{2} z^{2}\right] \\
& \quad a, k>0, x \geq \ln k, \omega_{1}, \omega_{2} \in[-1,1],-2 \leq \omega_{1}+\omega_{2} \leq 1
\end{aligned}
$$

and:

$$
\begin{aligned}
& f(x)=a k^{a} e^{-a x}\left[1-\omega_{1}-\omega_{2}+2\left(2 \omega_{2}+\omega_{1}\right) k^{a} e^{-a x}\right. \\
& \left.-3 \omega_{2} k^{2 a} e^{-2 a x}\right] \\
& =a z\left[1-\omega_{1}-\omega_{2}+2\left(2 \omega_{2}+\omega_{1}\right) z-3 \omega_{2} z^{2}\right]
\end{aligned}
$$

where, $a, k$ are a shape and location parameters respectively and $\omega_{1}, \omega_{2}$ are transmuted parameters.

Using Remark 1, Equation (17) can be rewritten in term of the random variable $Z$ as follows:

$$
\begin{aligned}
& f(z)=f[\varpi(z)] \frac{d x}{d z} \\
& =a z\left[1-\omega_{1}-\omega_{2}+2\left(2 \omega_{2}+\omega_{1}\right) z-3 \omega_{2} z^{2}\right]\left(\frac{-1}{a z}\right) \\
& =(-1)\left[1-\omega_{1}-\omega_{2}+2\left(2 \omega_{2}+\omega_{1}\right) z-3 \omega_{2} z^{2}\right] \\
& \text { if } z \in[0,1]
\end{aligned}
$$

\section{Proof}

The proof is straightforward. Equation (16) is obtained by substituting Equation (8) into Equation (6) and performing some algebra calculations while Equation (17) is gotten from differentiating Equation (16) with respect to $x$.

\section{Lemma 4}

The limit of CTEP-1-R density as $x \rightarrow \ln k$ is $a\left(1+\omega_{1}\right)$ and as $x \rightarrow \infty$ is 0 .

\section{Proof}

See proof of Lemma 1.

\section{Lemma 5}

$f(x)$ of Equation (17) is a probability density function.

\section{Proof}

To prove $f(x)$ is a pdf, we need to prove $f(x) \geq 0$ and $\int_{\ln k}^{\infty} f(x) d x=1$.

From Lemma (4) $\lim _{x \rightarrow \ln k} f(x)=a\left(1+\omega_{1}\right)$ and $\lim _{x \rightarrow \infty} f(x)=0$, since $a, k>0$ and $\omega_{1} \in[-1,1]$ then $a\left(1+\omega_{1}\right) \geq 0$. It follows that $f(x) \geq 0$.

The proof of $\int_{\ln k}^{\infty} f(x) d x=1$ is as follows:

$$
\begin{aligned}
& \int_{\ln k}^{\infty} f(x)=\int_{0}^{1} f(z) d x \\
& =\int_{0}^{1}\left[1-\omega_{1}-\omega_{2}+2\left(2 \omega_{2}+\omega_{1}\right) z-3 \omega_{2} z^{2}\right] d x \\
& =\left(1-\omega_{1}-\omega_{2}\right) \int_{0}^{1} d z+2\left(2 \omega_{2}+\omega_{1}\right) \int_{0}^{1} z d x \\
& -3 \omega_{2} \int_{0}^{1} z^{2} d z \\
& =1-\omega_{1}-\omega_{2}+\left(2 \omega_{2}+\omega_{1}\right)-\omega_{2}=1
\end{aligned}
$$

This completes the proof of Lemma.

Some of possible shapes of the pdf and cdf of CTEP1-R for different choices of parameters $\omega_{1}$ and $\omega_{2}$ setting $a=0.5$ and $k=1$ are illustrated in Fig. $3 \mathrm{a}$ and $3 \mathrm{~b}$ respectively. Note that the pdf and cdf of the base distribution EP-1 are shown as a solid black curves.

\section{Survival and Hazard Function}

The survival and hazard functions for CTEP-1-R distribution are given below:

$$
\begin{aligned}
& S(x)=1-(1-z)\left[1+\left(\omega_{1}+\omega_{2}\right) z-\omega_{2} z^{2}\right] \\
& h(x)=\frac{a z\left[1-\omega_{1}-\omega_{2}+2\left(2 \omega_{2}+\omega_{1}\right) z-3 \omega_{2} z^{2}\right]}{1-(1-z)\left[1+\left(\omega_{1}+\omega_{2}\right) z-\omega_{2} z^{2}\right]}
\end{aligned}
$$

where, $z=k^{a} e^{-a x}$.

Figures $4 \mathrm{a}$ and $4 \mathrm{~b}$ show the behavior of the survival and hazard functions of CTEP-1-R for the different choices of parameters $\omega_{1}$ and $\omega_{2}$ setting $a=0.5$ and $k=1$, where $s(x)$ and $h(x)$ of EP-1 distribution are shown as a solid black curves. Note that $h(x)$ for EP-1 distribution is a constant function, that is $h(x)=a$ if $\omega_{1}=\omega_{2}=0$. 


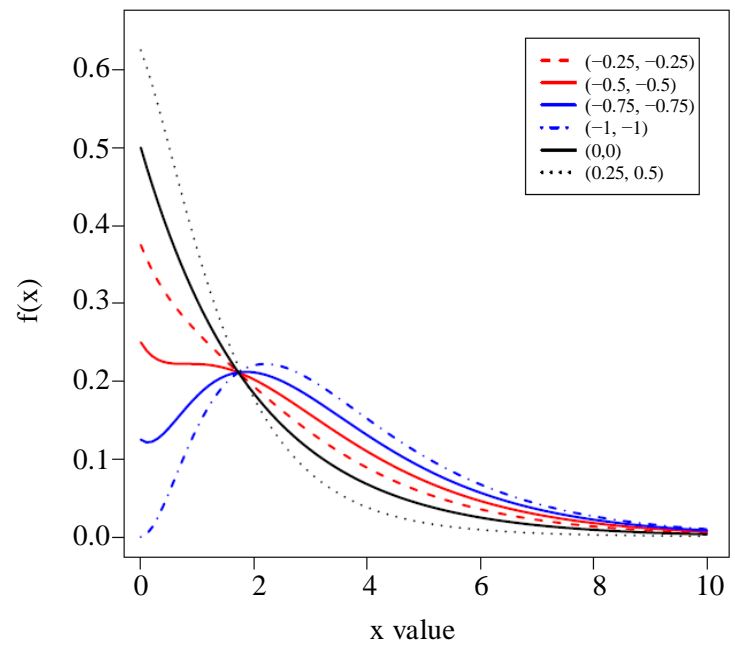

(a)

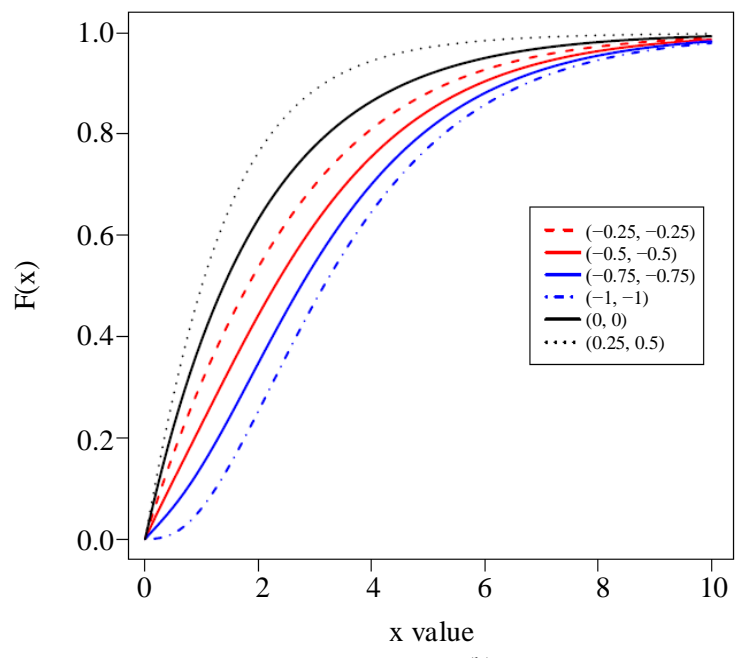

(b)

Fig. 3: The pdf and cdf of CTEP-1-R for different values of parameter $\lambda_{1}$ and $\lambda_{2}$ setting $a=0.5$ and $k=1$

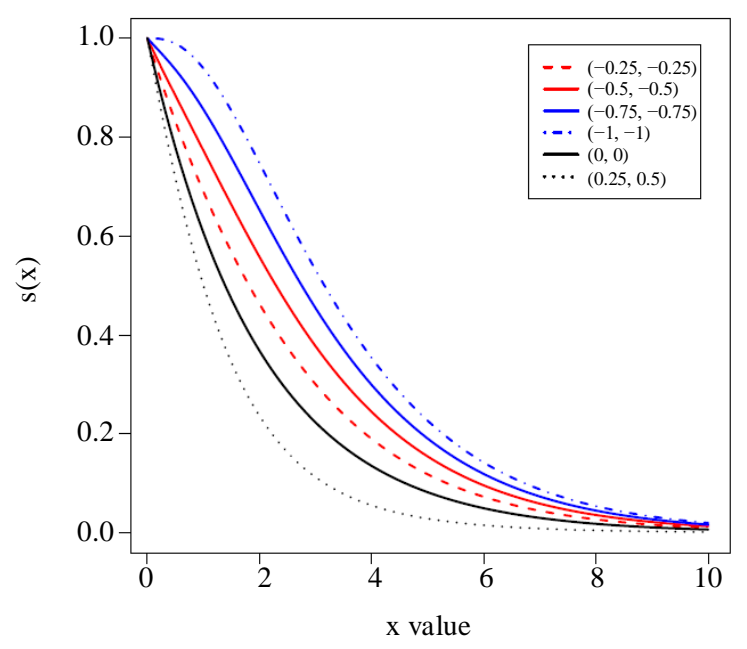

(a)

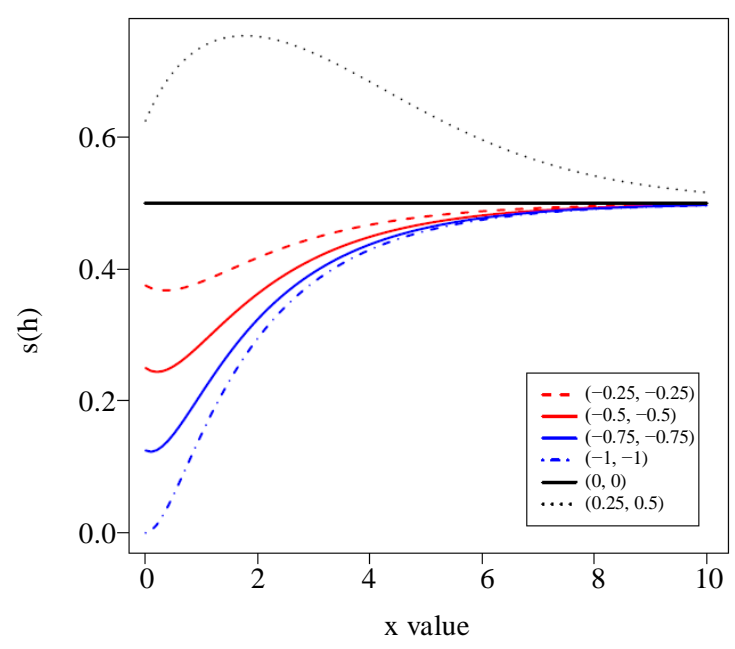

(b)

Fig. 4: The $s(x)$ and $h(x)$ of CTEP-1-R for different values of parameters $\lambda_{1}$ and $\lambda_{2}$ setting $a=0.5$ and $k=1$

\section{Statistical Properties}

In this subsection, some statistical properties for CTEP-1-R distribution are explained. These properties include moments and moment generating function.

\section{Moments}

\section{Theorem 5}

If $X$ is the random variable having the CTEP-1-R then the $r$ th moment of $X$ about the origin is:

$$
\begin{aligned}
& E\left(X^{r}\right)=\frac{k^{a}}{a^{r}}\left\{\left(1-\omega_{1}-\omega_{2}\right) \Gamma(r+1, a \ln k)\right. \\
& \left.+\frac{k^{a}}{2^{r}}\left(2 \omega_{2}+\omega_{1}\right) \Gamma(r+1,2 a \ln k)+\frac{k^{2 a}}{3^{r}} \omega_{2} \Gamma(r+1,3 a \ln k)\right\}
\end{aligned}
$$

\section{Proof}

See proof of Theorem 2 .

\section{Moments Generating Function}

\section{Theorem 6}

If $X$ is a random variable having the CTEP-1-R then the moments generating function of $X$ is:

$$
M_{X}(t)=\frac{a k^{t}\left(1-\omega_{1}-\omega_{2}\right)}{a-t}+\frac{2 a k^{t}\left(2 \omega_{2}+\omega_{1}\right)}{2 a-t}-\frac{3 a k^{t} \omega_{2}}{3 a-t}
$$

where, $a-t>0,2 a-t>0$ and $3 a-t>0$.

Further, the mean and variance of the distribution are given respectively as: 


$$
E(X)=\ln k+\frac{6-3 \omega_{1}-2 \omega_{2}}{6 a}
$$

and:

$$
\operatorname{Var}(X)=\frac{36-18 \omega_{1}-20 \omega_{2}-\left(3 \omega_{1}+2 \omega_{2}\right)^{2}}{36 a^{2}}
$$

\section{Proof}

See proof of Theorem 3.

The mean and variance have been abstracted from differentiating Equation (19) $i$ times $(i=1,2)$ with respect to $t$ and setting $t=0$. Table 3 and 4 explain the mean and variance of CTEP-1-R for various combinations of model parameters respectively.

From Table 3 and 4 it is observed that, holding the transmuted parameters $\omega_{1}$ and $\omega_{2}$ constants, as the shape parameter $a$ increases the mean and variance decrease. On the other hand, holding $a$ and $\omega_{1}$ constants, as $\omega_{2}$ increases the mean and variance are also decrease.

\section{Parameters Estimation}

This subsection discusses the Maximum Likelihood Estimation (MLE) for Parameters of CTEP-1-R distribution.

Let $X_{1}, X_{2}, \ldots, X_{n}$ be a random sample of size $n$ from CTEP-1-R distribution. Then the likelihood and $\log$ likelihood functions are given respectively by:

$$
\begin{aligned}
& L=a^{n} k^{a n} e^{-a \sum_{i=1}^{n} x_{i}} \prod_{i=1}^{n}\left[1-\omega_{1}-\omega_{2}+2\left(2 \omega_{2}+\omega_{1}\right)\right. \\
& \left.\times k^{a} e^{-a x_{i}}-3 \omega_{2} k^{2 a} e^{-2 a x_{i}}\right]
\end{aligned}
$$

and:

$$
\begin{aligned}
& \ln L=n \ln a+a n \ln k-a \sum_{i=1}^{n} x_{i}+\sum_{i=1}^{n} \ln \left[1-\omega_{1}-\omega_{2}\right. \\
& \left.+2\left(2 \omega_{2}+\omega_{1}\right) k^{a} e^{-a x_{i}}-3 \omega_{2} k^{2 a} e^{-2 a x_{i}}\right]
\end{aligned}
$$

The MLE of $\ln k$ is the first-order statistic $x_{(1)}$. The maximum likelihood estimates of $a, \omega_{1}$ and $\omega_{2}$ are obtained by maximize Equation (20). The derivatives of Equation (20) with respect to the unknown parameters are:

$$
\begin{aligned}
\frac{\partial \ln k}{\partial a}=\frac{n}{a}+n \ln k-\sum_{i=1}^{n} x_{i}+2 k^{a} \\
\times \sum_{i=1}^{n} \frac{\left(\ln k-x_{i}\right)\left[2 \omega_{2}+\omega_{1}+3 \omega_{2} k^{a} e^{-a x_{i}}\right] e^{-a x_{i}}}{R_{2}} \\
\frac{\partial \ln k}{\partial \omega_{1}}=\sum_{i=1}^{n} \frac{\left(2 k^{a} e^{-a x_{i}}-1\right)}{R_{2}} \\
\frac{\partial \ln k}{\partial \omega_{2}}=\sum_{i=1}^{n} \frac{\left[k^{a}\left(4-3 k^{a} e^{-a x_{i}}\right) e^{-a x_{i}}-1\right]}{R_{2}}
\end{aligned}
$$

\begin{tabular}{|c|c|c|c|c|c|c|}
\hline & & $\omega_{1}=-1$ & $\omega_{1}=-0.5$ & $\omega_{1}=0$ & $\omega_{1}=0.5$ & $\omega_{1}=1$ \\
\hline \multirow[t]{5}{*}{$a=1$} & $\omega_{2}=-1$ & 2.2388 & 1.9888 & 1.7388 & 1.4888 & 1.2388 \\
\hline & $\omega_{2}=-0.5$ & 2.0721 & 1.8221 & 1.5721 & 1.3221 & 1.0721 \\
\hline & $\omega_{2}=0$ & 1.9055 & 1.6555 & 1.4055 & 1.1555 & 0.9055 \\
\hline & $\omega_{2}=0.5$ & 1.7388 & 1.4888 & 1.2388 & 0.9888 & - \\
\hline & $\omega_{2}=1$ & 1.5721 & 1.3221 & 1.0721 & - & - \\
\hline \multirow[t]{5}{*}{$a=3$} & $\omega_{2}=-1$ & 1.0166 & 0.9332 & 0.8499 & 0.7666 & 0.6832 \\
\hline & $\omega_{2}=-0.5$ & 0.9610 & 0.8777 & 0.7944 & 0.7110 & 0.6277 \\
\hline & $\omega_{2}=0$ & 0.9055 & 0.8221 & 0.7388 & 0.6555 & 0.5721 \\
\hline & $\omega_{2}=0.5$ & 0.8499 & 0.7666 & 0.6832 & 0.5999 & - \\
\hline & $\omega_{2}=1$ & 0.7944 & 0.7110 & 0.6277 & - & - \\
\hline \multirow[t]{5}{*}{$a=5$} & $\omega_{2}=-1$ & 0.7721 & 0.7221 & 0.6721 & 0.6221 & 0.5721 \\
\hline & $\omega_{2}=-0.5$ & 0.7388 & 0.6888 & 0.6388 & 0.5888 & 0.5388 \\
\hline & $\omega_{2}=0$ & 0.7055 & 0.6555 & 0.6055 & 0.5555 & 0.5055 \\
\hline & $\omega_{2}=0.5$ & 0.6721 & 0.6221 & 0.5721 & 0.5221 & - \\
\hline & $\omega_{2}=1$ & 0.6388 & 0.5888 & 0.5388 & - & - \\
\hline
\end{tabular}

where:

$$
R_{2}=\left[1-\omega_{1}-\omega_{2}+2\left(2 \omega_{2}+\omega_{1}\right) k^{a} e^{-a x_{i}}-3 \omega_{2} k^{2 a} e^{-2 a x_{i}}\right] .
$$

Now setting, $\frac{\partial \ln k}{\partial a}=0 \frac{\partial \ln k}{\partial \lambda_{1}}=0$ and $\frac{\partial \ln k}{\partial \lambda_{2}}=0$ and solving the nonlinear system of equations, the maximum likelihood estimates $\hat{\theta}=\left(\hat{a}, \hat{\omega}_{1}, \hat{\omega}_{2}\right)$ of $\theta=$ $\left(a, \omega_{1}, \omega_{2}\right)$ is obtained.

Table 3: Mean of CTEP-1-R distribution 
Hussein Eledum / Journal of Mathematics and Statistics 2020, 16: 113.124 DOI: 10.3844/jmssp.2020.113.124

Table 4: Variance of CTEP-1-R distribution

\begin{tabular}{|c|c|c|c|c|c|c|}
\hline & & $\omega_{1}=-1$ & $\omega_{1}=-0.5$ & $\omega_{1}=0$ & $\omega_{1}=0.5$ & $\omega_{1}=1$ \\
\hline \multirow[t]{5}{*}{$a=1$} & $\omega_{2}=-1$ & 1.3611 & 1.4653 & 1.3611 & 1.2986 & 1.0278 \\
\hline & $\omega_{2}=-0.5$ & 1.3333 & 1.3542 & 1.3333 & 1.0208 & 0.6667 \\
\hline & $\omega_{2}=0$ & 1.2500 & 1.1875 & 1.2500 & 0.6875 & 0.2500 \\
\hline & $\omega_{2}=0.5$ & 1.1111 & 0.9653 & 1.1111 & 0.2986 & - \\
\hline & $\omega_{2}=1$ & 0.9167 & 0.6875 & 0.9167 & - & - \\
\hline \multirow[t]{5}{*}{$a=3$} & $\omega_{2}=-1$ & 0.1512 & 0.1628 & 0.1605 & 0.1443 & 0.1142 \\
\hline & $\omega_{2}=-0.5$ & 0.1481 & 0.150 & 0.1389 & 0.1134 & 0.0741 \\
\hline & $\omega_{2}=0$ & 0.1389 & 0.1319 & 0.1111 & 0.0764 & 0.0278 \\
\hline & $\omega_{2}=0.5$ & 0.1235 & 0.1073 & 0.0772 & 0.0332 & - \\
\hline & $\omega_{2}=1$ & 0.1019 & 0.0764 & 0.0370 & - & - \\
\hline \multirow[t]{5}{*}{$a=5$} & $\omega_{2}=-1$ & 0.0544 & 0.0586 & 0.0578 & 0.0519 & 0.0411 \\
\hline & $\omega_{2}=-0.5$ & 0.0533 & 0.0542 & 0.0500 & 0.0408 & 0.0267 \\
\hline & $\omega_{2}=0$ & 0.0500 & 0.0475 & 0.0400 & 0.0275 & 0.0100 \\
\hline & $\omega_{2}=0.5$ & 0.0444 & 0.0386 & 0.0278 & 0.0119 & - \\
\hline & $\omega_{2}=1$ & 0.0367 & 0.0275 & 0.0133 & - & - \\
\hline
\end{tabular}

Table 5: Kevlar 49/epoxy strands failure times (pressure at 90\%) (FTK) dataset

\begin{tabular}{|c|c|c|c|c|c|c|c|c|c|c|c|c|c|c|}
\hline 0.01 & 0.01 & 0.02 & 0.02 & 0.02 & 0.03 & 0.03 & 0.04 & 0.05 & 0.06 & 0.07 & 0.07 & 0.08 & 0.09 & 0.09 \\
\hline 0.1 & 0.1 & 0.11 & 0.11 & 0.12 & 0.13 & 0.18 & 0.19 & 0.2 & 0.23 & 0.24 & 0.24 & 0.29 & 0.34 & 0.35 \\
\hline 0.36 & 0.38 & 0.4 & 0.42 & 0.43 & 0.52 & 0.54 & 0.56 & 0.6 & 0.6 & 0.63 & 0.65 & 0.67 & 0.68 & 0.72 \\
\hline 0.72 & 0.72 & 0.73 & 0.79 & 0.79 & 0.8 & 0.8 & 0.83 & 0.85 & 0.9 & 0.92 & 0.95 & 0.99 & 1 & 1.01 \\
\hline 1.02 & 1.03 & 1.05 & 1.1 & 1.1 & 1.11 & 1.15 & 1.18 & 1.2 & 1.29 & 1.31 & 1.33 & 1.34 & 1.4 & 1.43 \\
\hline 1.45 & 1.5 & 1.51 & 1.52 & 1.53 & 1.54 & 1.54 & 1.55 & 1.58 & 1.6 & 1.63 & 1.64 & 1.8 & 1.8 & 1.81 \\
\hline 2.02 & 2.05 & 2.14 & 2.17 & 2.33 & 3.03 & 3.03 & 3.34 & 4.2 & 4.69 & 9.89 & & & & \\
\hline
\end{tabular}

Table 6: Failure Times of Components (FTC) dataset

\begin{tabular}{|c|c|c|c|c|c|c|c|c|c|c|c|c|}
\hline 0.008 & 0.017 & 0.058 & 0.061 & 0.084 & 0.090 & 0.134 & 0.238 & 0.245 & 0.353 & 0.374 & 0.480 & 0.495 \\
\hline 0.535 & 0.564 & 0.681 & 0.686 & 0.688 & 0.921 & 0.959 & 1.022 & 1.092 & 1.260 & 1.284 & 1.295 & 1.373 \\
\hline 1.395 & 1.414 & 1.760 & 1.858 & 1.892 & 1.921 & 1.926 & 1.933 & 2.135 & 2.169 & 2.301 & 2.320 & 2.405 \\
\hline 2.506 & 2.598 & 2.808 & 2.971 & 3.087 & 3.492 & 3.669 & 3.926 & 4.446 & 5.119 & 8.596 & & \\
\hline
\end{tabular}

Table 7: Summary statistics for selected datasets

\begin{tabular}{llllllll}
\hline & $n$ & Min & Max & Mean & Median & Skewness & Kurtosis \\
\hline FTK & 101 & 0.01 & 9.890 & 1.0447 & 0.8 & 4.0704 & 24.9668 \\
FTC & 50 & 0.008 & 8.596 & 1.6728 & 1.33 & 1.9165 & 08.4351 \\
\hline
\end{tabular}

Table 8: Parameters estimates, -Log (L), k-s test value and p-value for the selected distributions for FTK dataset

\begin{tabular}{|c|c|c|c|c|c|c|}
\hline Distribution & \multicolumn{3}{|c|}{ Parameters estimates } & $-\log (\mathrm{L})$ & $\mathrm{k}-\mathrm{s}$ & P-value \\
\hline EP-1 & $a=0.9665$ & & & 104.441 & 0.0984 & 0.282 \\
\hline CTEP-1 & $a=0.9444$ & $\lambda=0.1887$ & & 104.1523 & 0.08466 & 0.4641 \\
\hline CTEP-1-G & $a=1.3989$ & $\lambda_{1}=0.99$ & $\lambda_{2}=-0.3735$ & 102.2592 & 0.0824 & 0.4987 \\
\hline CTEP-1-R & $a=0.56539$ & $\omega_{1}=0.81430$ & $\omega_{2}=0.05022$ & 103.2035 & 0.0922 & 0.3569 \\
\hline
\end{tabular}

Table 9: Parameters estimates, -log (L), k-s test value and p-value for the selected distributions for FTC dataset

\begin{tabular}{|c|c|c|c|c|c|c|}
\hline Distribution & \multicolumn{3}{|c|}{ Parameters estimates } & $-\log (\mathrm{L})$ & $\mathrm{k}-\mathrm{s}$ & P-value \\
\hline EP-1 & $a=0.6006$ & & & 75.4877 & 0.0909 & 0.7694 \\
\hline CTEP-1 & $a=0.5983$ & $\lambda=0.0336$ & & 75.4832 & 0.0921 & 0.7552 \\
\hline CTEP-1-G & $a=0.8577$ & $\lambda_{1}=0.99$ & $\lambda_{2}=-0.296$ & 74.0117 & 0.061214 & 0.9863 \\
\hline CTEP-1-R & $a=0.3344$ & $\omega_{1}=0.6582$ & $\omega_{2}=0.3418$ & 75.3877 & 0.08412 & 0.8419 \\
\hline
\end{tabular}




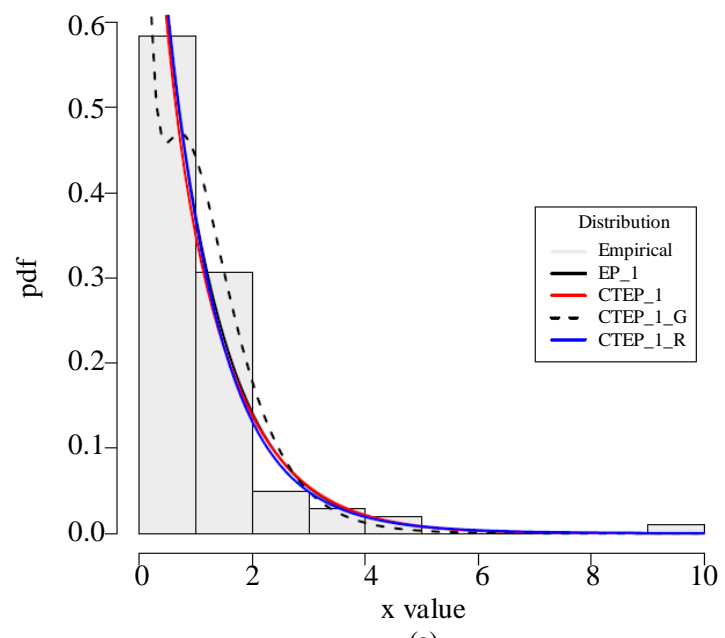

(a)

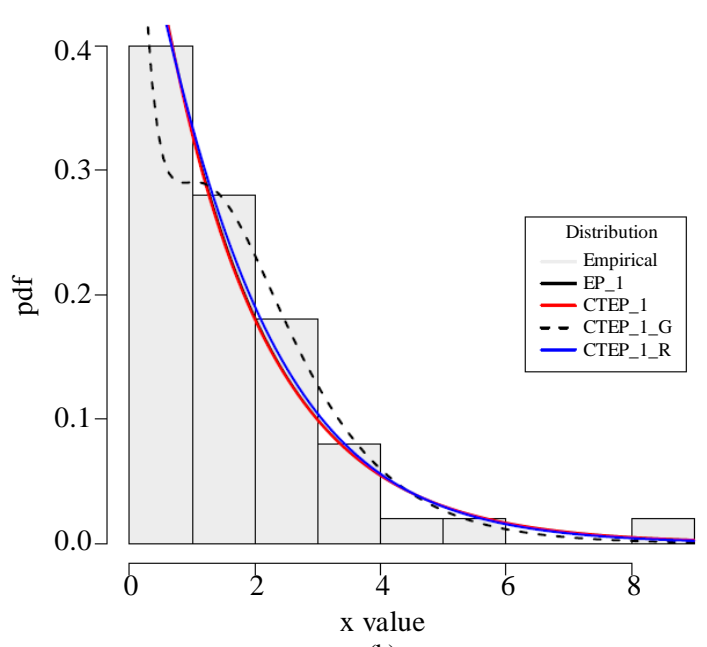

(b)

\section{Application of CTEP-1-G and CTEP-1-R distributions}

In this section the proposed distributions CTEP-1-G and CTEP-1-R are fitted to two real lifetime datasets to test their goodness of fit. Moreover, CTEP-1-G and CTEP-1-R are compared with some related distributions included the exponentiated Pareto-I (EP-I) and cubic transmuted exponentiated Pareto-I (CTEP-I) distributions. With a view to compare CTEP-1-G and CTEP-1-R some different comparison measures includes -log-likelihood and Kolmogorov-Smirnov $(k-s)$ test are used. For the aim of the analysis we set $\ln k=$ $\exp [\min (x)]$. The computations are done by $R$.

The first data in Table 5 is leptokurtic, bimodal, highly right skewed reported by (Barlow et al., 1984) represents the failure times of Kevlar 49/epoxy strands when the pressure is at $90 \%$ stress level (FTK) and used by (Andrews and Herzberg, 2012; Cooray and Ananda, 2008; Al-Aqtash et al., 2014; Ansari and Eledum, 2020).

The second data in Table 6 is leptokurtic bimodal, moderate right skewed adduced by (Murthy et al., 2004) shows the Failure Times (in hours) of 50 Components (FTC).

Table 7 shows the summary statistics of the two datasets. Tables 8 and 9 respectively demonstrate the results obtained from the two datasets including maximum likelihood estimates and comparison measures for the fitted distributions. Moreover, the estimated pdf's and cdf's of (FTK) and (FTC) are displayed in Fig. 5a and $5 \mathrm{~b}$ respectively.

Based on the values of the k-s and its corresponding p-values in Table 8 we observe that all distributions fit the Kevlar 49/epoxy strands failure times data adequacy while the CTEP-1-G is the best one Fig. 5a. Moreover, CTEP-1-G has the minimum value of $-\log (\mathrm{L})(101.231)$. We conclude that the CTEP-1-G is the most appropriate model for FTK dataset.
From the results of FTC dataset in Table 9 it is clear that all distributions fit the Failure Times of components data adequacy while the CTEP-1-G is the best one Fig. 5b and it has the minimum value of $-\log (\mathrm{L})$ (74.017).

Hence we infer that the CTEP-1-G is the most appropriate model for FTC dataset.

\section{Conclusion}

In this study two new generalized models of the Exponentiated Pareto-I distribution Called (CTEP-I-G) and (CTEP-I-R) are introduced. Some statistical properties of the two distributions including survival and hazard functions, moments and moment generating function are studied. The models parameters are estimated by using the maximum likelihood method. Finally, an application of CTEP-I-G and CTEP-I-R to two real datasets and compared with the exponentiated Pareto-I and cubic transmuted exponentiated Pareto-1 distributions is illustrated. Based on applications results we conclude that the proposed distributions CTEP-I-G and CTEP-I$\mathrm{R}$ have fitted the two datasets better than other distributions, further among the proposed distributions the CTEP-I-G is the best. We recommend the proposed distributions for modelling the failure times datasets adequacy and hope that they would receive significant applications in the future.

\section{Acknowledgement}

The author is grateful to the editor and the anonymous reviewers for their valuable comments and suggestions, which have substantially improved this paper.

\section{Ethics}

This article is original and contains unpublished material and no ethical issues involved. 


\section{References}

Al-Aqtash, R., Lee, C., \& Famoye, F. (2014). GumbelWeibull distribution: Properties and applications. Journal of Modern applied statistical methods, 13(2), 11

Alizadeh, M., Merovci, F., \& Hamedani, G. G. (2016). Generalized transmuted family of distributions: Properties and applications. Hacettepe University Bulletin of Natural Sciences and Engineering Series B: Mathematics and Statistics.

AL-Kadim, K. A., \& Mohammed, M. H. (2017). The cubic transmuted Weibull distribution. Journal of University of Babylon, 3, 862-876.

AL-Kadim, K. A. (2018). Proposed Generalized Formula for Transmuted Distribution. Journal of University of Babylon for Pure and Applied Sciences, 26(4), 66-74.

Andrews, D. F., \& Herzberg, A. M. (2012). Data: a collection of problems from many fields for the student and research worker. Springer Science \& Business Media.

Ansari, S. I., \& Eledum, H. (2018). An Extension of Pareto Distribution. Journal of Statistics Applications \& Probability, 7, 443-455.

Ansari, S.I. and Eledum, H. (2020). A new generalization of exponentiated Pareto-I distribution. Journal Modern Applied Stat. Meth.

Aryal, G. R., \& Tsokos, C. P. (2009). On the transmuted extreme value distribution with application. Nonlinear Analysis: Theory, Methods \& Applications, 71(12), e1401-e1407.

Aryal, G. R., \& Tsokos, C. P. (2011). Transmuted Weibull distribution: A generalization of theWeibull probability distribution. European Journal of Pure and Applied Mathematics, 4(2), 89-102.

Barlow, R. E., Toland, R. H., \& Freeman, T. (1984). A Bayesian analysis of stress-rupture life of kevlar 49/epoxy spherical pressure vessels. In Proc. Conference on Applications of Statistics', Marcel Dekker, New York.

Celik, N. (2018). Some Cubic Rank Transmuted Distributions. Journal of Applied Mathematics, Statistics and Informatics, 14(2), 27-43.

Cooray, K., \& Ananda, M. M. (2008). A generalization of the half-normal distribution with applications to lifetime data. Communications in Statistics-Theory and Methods, 37(9), 1323-1337.

Cordeiro, G. M., \& de Castro, M. (2011). A new family of generalized distributions. Journal of statistical computation and simulation, 81(7), 883-898.

Eugene, N., Lee, C., \& Famoye, F. (2002). Beta-normal distribution and its applications. Communications in Statistics-Theory and methods, 31(4), 497-512.
Granzotto, D. C. T., Louzada, F., \& Balakrishnan, N. (2017). Cubic rank transmuted distributions: Inferential issues and applications. Journal of statistical Computation and Simulation, 87(14), 2760-2778.

Gupta, R. C., Gupta, P. L., \& Gupta, R. D. (1998). Modeling failure time data by Lehman alternatives. Communications in Statistics-Theory and methods, 27(4), 887-904.

Gupta, R. D., \& Kundu, D. (1999). Theory \& methods: Generalized exponential distributions. Australian \& New Zealand Journal of Statistics, 41(2), 173-188.

Gupta, R. D., \& Kundu, D. (2001). Exponentiated exponential family: An alternative to gamma and Weibull distributions. Biometrical Journal: Journal of Mathematical Methods in Biosciences, 43(1), 117-130.

Gupta, R. D., \& Kundu, D. (2007). Generalized exponential distribution: Existing results and some recent developments. Journal of Statistical Planning and Inference, 137(11), 3537-3547.

Merovci, F., Alizadeh, M., \& Hamedani, G. G. (2016). Another generalized transmuted family of distributions: Properties and applications. Austrian Journal of Statistics, 45(3), 71-93.

Murthy, D.P., Xie M. and Jiang, R. (2004). Exponentiated pare to distributions. Statistics, 39, 255-260.

Nadarajah, S. (2005). Exponentiated Pareto distributions. Statistics, 39(3), 255-260.

Nofal, Z. M., Afify, A. Z., Yousof, H. M., \& Cordeiro, G. M. (2017). The generalized transmuted-G family of distributions. Communications in Statistics-Theory and Methods, 46(8), 4119-4136.

Rahman, M. M., Al-Zahrani, B., \& Shahbaz, M. Q. (2018a). Cubic Transmuted Pareto Distribution. Annals of Data Science, 1-18.

Rahman, M. M., Al-Zahrani, B., \& Shahbaz, M. Q. (2018b). A general transmuted family of distributions. Pakistan Journal of Statistics and Operation Research, 451-469.

Rahman, M. M., Al-Zahrani, B., \& Shahbaz, M. Q. (2019). Cubic transmuted Weibull distribution: Properties and applications. Annals of Data Science, 6(1), 83-102.

Shaw, W. T., \& Buckley, I. R. (2009). The alchemy of probability distributions: Beyond Gram-Charlier expansions and a skew-kurtotic-normal distribution from a rank transmutation map. arXiv preprint arXiv:0901.0434. 